ARTICLE

Received 9 May 2015 | Accepted 4 Dec 2015 | Published 22 Jan 2015

DOI: $10.1038 /$ ncomms10364

OPEN

\title{
Dopant-specific unzipping of carbon nanotubes for intact crystalline graphene nanostructures
}

Joonwon Lim¹, Uday Narayan Maiti', Na-Young Kim², Rekha Narayan', Won Jun Lee , Dong Sung Choi', Youngtak Oh${ }^{1}$, Ju Min Lee ${ }^{1}$, Gil Yong Lee', Seok Hun Kang ${ }^{1}$, Hyunwoo Kim³ ${ }^{3}$ Yong-Hyun Kim² \& Sang Ouk Kim ${ }^{1}$

Atomic level engineering of graphene-based materials is in high demand to enable customize structures and properties for different applications. Unzipping of the graphene plane is a potential means to this end, but uncontrollable damage of the two-dimensional crystalline framework during harsh unzipping reaction has remained a key challenge. Here we present heteroatom dopant-specific unzipping of carbon nanotubes as a reliable and controllable route to customized intact crystalline graphene-based nanostructures. Substitutional pyridinic nitrogen dopant sites at carbon nanotubes can selectively initiate the unzipping of graphene side walls at a relatively low electrochemical potential $(0.6 \mathrm{~V})$. The resultant nanostructures consisting of unzipped graphene nanoribbons wrapping around carbon nanotube cores maintain the intact two-dimensional crystallinity with well-defined atomic configuration at the unzipped edges. Large surface area and robust electrical connectivity of the synergistic nanostructure demonstrate ultrahigh-power supercapacitor performance, which can serve for AC filtering with the record high rate capability of $-85^{\circ}$ of phase angle at $120 \mathrm{~Hz}$.

\footnotetext{
${ }^{1}$ National Creative Research Initiative Center for Multi-Dimensional Directed Nanoscale Assembly, Department of Materials Science and Engineering, KAIST, Daejeon 34141, Republic of Korea. ${ }^{2}$ Graduate School of Nanoscience and Technology, KAIST, Daejeon 34141, Republic of Korea. ${ }^{3}$ Department of Chemistry, KAIST, Daejeon 34141, Republic of Korea. Correspondence and requests for materials should be addressed to Y.-H.K. (email: yong.hyun.kim@kaist.ac.kr) or to S.O.K. (email: sangouk.kim@kaist.ac.kr).
} 
nzipping of graphene basal plane is a valuable route to tailor carbon nanostructures ${ }^{1-9}$. Complete nanoscale unzipping of carbon nanotubes (CNTs) or graphene may produce graphene nanoribbons with electrical energy band gap $^{10-14}$. Partial unzipping of CNTs may create unique nanostructures where CNTs and graphene nanoribbons are seamlessly connected ${ }^{2,15}$. Since the pioneering work by Tour et al. ${ }^{1}$, many different unzipping mechanisms have been explored. Wet chemical methods, including chemical oxidation ${ }^{1,16}, \mathrm{Li}$ ion intercalation $^{17}$, sonication ${ }^{18}$, electrochemical method ${ }^{19}$ and hydrothermal reaction ${ }^{20}$ are advantageous for scalable solution processing. Dry processing techniques, such as plasma etching ${ }^{12}$, metal particle catalytic cutting ${ }^{21}$, rapid thermal expansion ${ }^{22}$ and high mechanical impact ${ }^{23}$ can reduce the oxidative degradation of unzipped structures. Unfortunately, harsh reaction conditions for the unzipping of robust $\mathrm{sp}^{2}$ hybridized graphene plane commonly accompanies undesired damage in the basal plane. A better controllability over unzipping mechanism is highly demanded for the minimal damage to the genuine graphene-based carbon structures ${ }^{16,24-27}$

In this work, we present dopant-specific electrochemical unzipping of CNTs as a controllable method for intact crystalline unzipping. Heteroatom dopants, such as nitrogen substitutionally incorporated into $\mathrm{sp}^{2}$ hybridized carbon framework, enable atomic-scale site-selective unzipping reaction. Although pristine CNTs are stable up to the electrochemical potential above $0.8 \mathrm{~V}$, nitrogen-doped CNTs (NCNTs) are readily unzipped below $0.6 \mathrm{~V}$. Detailed investigation on the reaction mechanism reveals that substitutional pyridinic nitrogen $\left(\mathrm{N}_{\mathrm{p}}\right)$-dopant can specifically initiate CNT wall unzipping. Such a dopant-specific unzipping at moderate potential enables fine controllability of unzipping level and intact crystallinity of the unzipped structures with well-defined edge configuration. Taking advantage of these unique features, we are able to synthesize intact crystalline graphene-based nanostructures, where unzipped graphene nanoribbons are seamlessly connected to the CNT strands. This structural feature offers synergistic properties comprising large surface area and robust electrical conductivity, which are highly desirable for electrochemical applications, such as energy storage. As a representative application, we demonstrate ultrahigh-power double-layer capacitors (DLCs) for alternating current (AC) line-filtering performance.

\section{Results}

Preparation and characterization of unzipped nanostructures. Figure 1 presents the carbon nanostructures produced from the electrochemical unzipping of NCNT forest. Unzipping procedure is schematically described in Fig. 1a. While NCNT forest is immersed in $1 \mathrm{M} \mathrm{H}_{2} \mathrm{SO}_{4}$ aqueous solution at a positive potential of $0.6-0.8 \mathrm{~V}$, electrochemically developed high-energy oxygen species react with the carbon atoms nearby the $\mathrm{N}_{\mathrm{p}}$-dopant sites and initiate unzipping ${ }^{24,28}$. As unzipping propagates at outermost walls, inner walls are exposed to $\mathrm{H}_{2} \mathrm{SO}_{4}$ solution and subsequently unzipped (Supplementary Fig. 1a). Eventually, the carbon nanostructures, consisting of unzipped graphene nanoribbons wrapping around NCNT core (Fig. 1a and Supplementary Fig. 1b,c) are formed, as confirmed by field-emission scanning electron microscopy. Close observation of the unzipped outer NCNT walls with aberration-corrected transmission electron microscopy (TEM) reveals the seamless junction between intact sidewalls and unzipped parts (Fig. 1b).

Figure 1c shows an aberration-corrected TEM image of the unzipped outmost CNT walls. Significantly, the basal plane of the unzipped graphene sheets maintains the intact crystallinity of parent NCNTs, as characterized by fast Fourier transform analysis. The configuration of edges developed during unzipping reaction is characterized with the angle between in-plane hexagonal carbon lattice and edge direction ${ }^{29,30}$ (Fig. 1d). Zigzag edge configuration (green line) predominates in the unzipped structures (Supplementary Fig. 2). Figure 1e shows an atomic-force microscopy (AFM) image of 1.7-nm-thick tri-layer graphene sheet produced from 5.9-nm-diameter triple-walled NCNTs (Supplementary Fig. 3). The lateral width of unzipped structures is slightly larger than the diameter of parent NCNTs as expected (Supplementary Fig. 4). After unzipping, solvent exchange with volatile alcohols and subsequent critical point drying restore the vertically aligned morphology (Supplementary Fig. 5).

Chemical structure of typical unzipped materials was characterized by X-ray photoelectron spectroscopy (XPS) and Raman spectroscopy. Deconvoluted C1s XPS spectrum (Fig. 1f) shows the five distinct peaks for graphitic structure $(\mathrm{C}-\mathrm{C} / \mathrm{C}=\mathrm{C}$ at $284.6 \mathrm{eV})$, carbon-nitrogen covalent bond $(\mathrm{C}-\mathrm{N}$ at $285.7 \mathrm{eV})$, hydroxyl/epoxy groups $(\mathrm{C}-\mathrm{O}$ at $286.6 \mathrm{eV})$, carbonyl group $(\mathrm{C}=\mathrm{O}$ at $287.8 \mathrm{eV})$ and carboxyl group $(\mathrm{O}-\mathrm{C}=\mathrm{O}$ at $288.7 \mathrm{eV})$, respectively. Substantial decrease of the $\mathrm{C}-\mathrm{N}$ peak intensity after unzipping signifies a specific chemical modification involved with $\mathrm{N}$-dopant sites. Appearance of the minor peaks for oxygen functional groups demonstrates a low level of oxidation during unzipping. In the Raman spectra (Fig. 1g), a moderate increase in the $G$ to $D$ peak intensity ratio $\left(I_{\mathrm{D}} / I_{\mathrm{G}}\right)$ from 1.06 to 1.52 and up-shift of $\mathrm{G}$ band position from 1,581 to $1,588 \mathrm{~cm}^{-1}$ result from the decrease of in-plane $\mathrm{sp}^{2}$ domain size upon $\mathrm{C}=\mathrm{C}$ bond breaking reactions $26,31-33$.

Reaction mechanism for $\mathbf{N}$-dopant-specific unzipping. The high-crystalline unzipping of NCNTs with low oxygen content implies a well-defined chemical reaction rather than violent random oxidative reactions. N1s XPS results of NCNTs before and after unzipping (Fig. 2a) reveal that $\mathrm{N}_{\mathrm{p}}$ peak is specifically reduced during unzipping, whereas quaternary nitrogen $\left(\mathrm{N}_{\mathrm{Q}}\right)$ is not influenced. Concurrently, Fe2p XPS results (Fig. 2b) indicate the disappearance of Fe species in the unzipped structures. In our plasma-enhanced chemical vapour deposition growth of NCNTs from $\mathrm{Fe}$ catalyst, one Fe atom taken from catalyst complexes with four $\mathrm{N}_{\mathrm{p}}$ atoms to generate $\mathrm{FeN}_{4}$ moieties in the graphene plane ${ }^{34}$ (Supplementary Table 1). In an acidic unzipping environment, two protons $\left(2 \mathrm{H}^{+}\right)$can replace the $\mathrm{Fe}^{2+}$ ion to form $\mathrm{H}_{2} \mathrm{~N}_{4}$ moiety (Supplementary Fig. 6 and Supplementary Note 1), which further undergoes acid-catalysed imine hydrolysis reaction (Supplementary Fig. 7). Protonation at the imine nitrogen forms iminium ion, which reacts with water followed by the proton transfer. This process could lead to the formation of carbonyl and hydroxyl groups along with the removal of $\mathrm{NH}_{3}$, as shown in Fig. $2 c$. The $N_{p}$ is thus eliminated from the graphitic plane, as verified by N1s XPS data.

To propose the detailed propagation mechanism for unzipping, we investigated on the reactive species for $\mathrm{C}-\mathrm{C}$ bond cleavage. Noteworthy, the presence of ozone molecules were detected under our electrochemical oxidative conditions (Fig. $2 \mathrm{~d}$ and Supplementary Fig. 8). The reactive ozone species preferentially attack the alkene in the $\alpha, \beta$-position of the carbonyl group, which is highly strained by the steric repulsion between carbonyl and hydroxyl groups. Moreover, the density functional theory (DFT) computation provides a probable reaction pathway for unzipping (Supplementary Fig. 9). It was found that the resulting ozonide formed from ozone and alkene can be dissociated into two molecules of carbonyl compounds and generate an epoxide from the next nearest strained alkene. This process can be repeated by the incorporation of singlet 
a
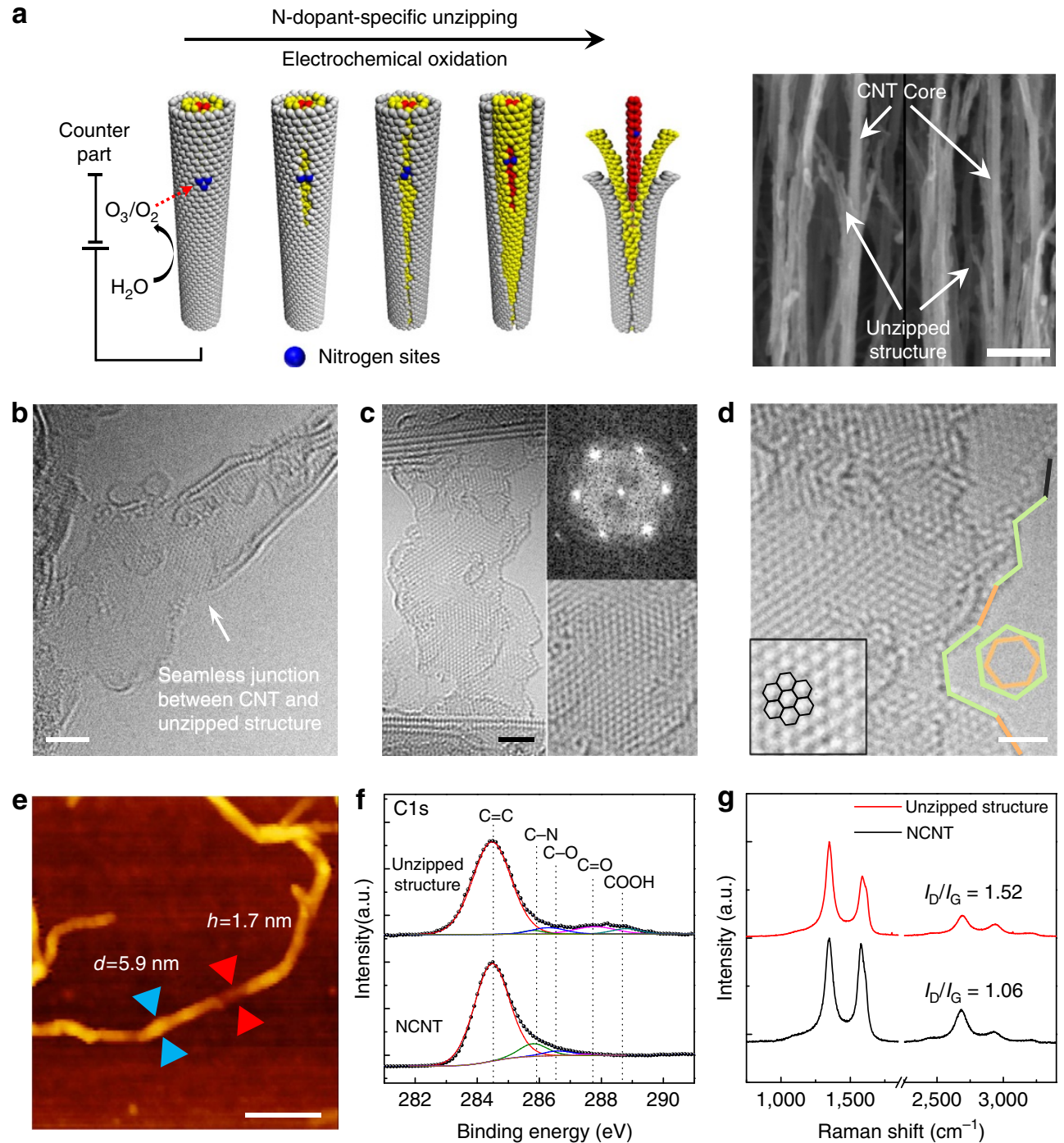

Figure 1 | Carbon nanostructure formation by $\mathbf{N}$-dopant-specific unzipping. (a) Schematic illustration of N-dopant-specific unzipping of NCNTs and scanning electron microscopy images of resulting unzipped nanostructures consisting of graphene nanoribbons wrapping around NCNT cores. (b-d) Aberration-corrected TEM images of unzipped nanostructures. (b) Seamless junction between CNT and unzipped nanoribbons. (c) Intact crystalline unzipped graphene nanoribbons with fast Fourier transformation analysis (top right) and magnified observation showing in-plain hexagonal carbon lattice (bottom right). (d) Unfurled unzipped edge with zigzag-predominant configuration. Green, orange and black colour indicate zigzag edges, armchair edges and chiral-edges, respectively. Inset shows in-plain hexagonal carbon lattice as an angle reference for edge-configuration characterization. (e) AFM image of unzipped nanostructure, where NCNTs and graphene nanoribbons are seamlessly connected. Blue and red arrows indicate NCNT and graphene nanoribbons, respectively. (f,g) C1s XPS and Raman spectra of unzipped nanostructures (top) and NCNTs (bottom).

Scale bars are $50 \mathrm{~nm}$ in $\mathbf{a}, 2 \mathrm{~nm}$ in $\mathbf{b}, \mathbf{c}, 1 \mathrm{~nm}$ in $\mathbf{d}$ and $100 \mathrm{~nm}$ in $\mathbf{e}$.

oxygen (Fig. 2d). This propagation mechanism explains the directional unzipping to occur via sequential generation of strained alkenes that act as the key oxidation sites due to the steric constraints imposed by the carbonyl groups (Supplementary Fig. 10). Consequently, unzipping propagates along the chemically favoured direction rather than random cutting 1,35 . Termination of the unzipping occurs when the inmost NCNT is exposed. Partial unzipping of the inmost NCNT, which is the final electrical pathway for electrochemical unzipping, leads to the serious degradation of electrical connectivity. Insufficient electrical potential delivery via the damaged inmost wall selfterminates the unzipping process (Supplementary Fig. 11). This rational suggestion for $\mathrm{N}_{\mathrm{p}}$ site-initiated unzipping mechanism provides excellent motivation for further study to fully resolve the role of heteroatom in unzipping reaction, including other possible heteroatom dopants.
Precise control of unzipping level. An essential requirement for the customized unzipping is the precise controllability of initiation, propagation and termination steps. In our approach, the density of $\mathrm{N}_{\mathrm{p}}$-dopants (unzipping initiation sites) is easily tunable by adjusting $\mathrm{NH}_{3}$ gas partial pressure during the synthesis of $\mathrm{NCNTs}^{36,37}$ (Supplementary Fig. 12). Electrical potential is another significant parameter for highly selective unzipping reactions ${ }^{19}$. Undoped CNTs are inert up to $0.8 \mathrm{~V}$ (Supplementary Fig. 13), above which random reaction occurs and severely damages the CNT surface ${ }^{38}$. By contrast, $\mathrm{N}_{\mathrm{p}}$-dopant-specific unzipping is triggered at the significantly reduced potential of $0.6 \mathrm{~V}$ (Supplementary Fig. 14). In this work, a moderate potential of $0.8 \mathrm{~V}$ is determined as the optimum potential value for a highly selective unzipping from $\mathrm{N}_{\mathrm{p}}$-dopant sites with manageable unzipping rate. 

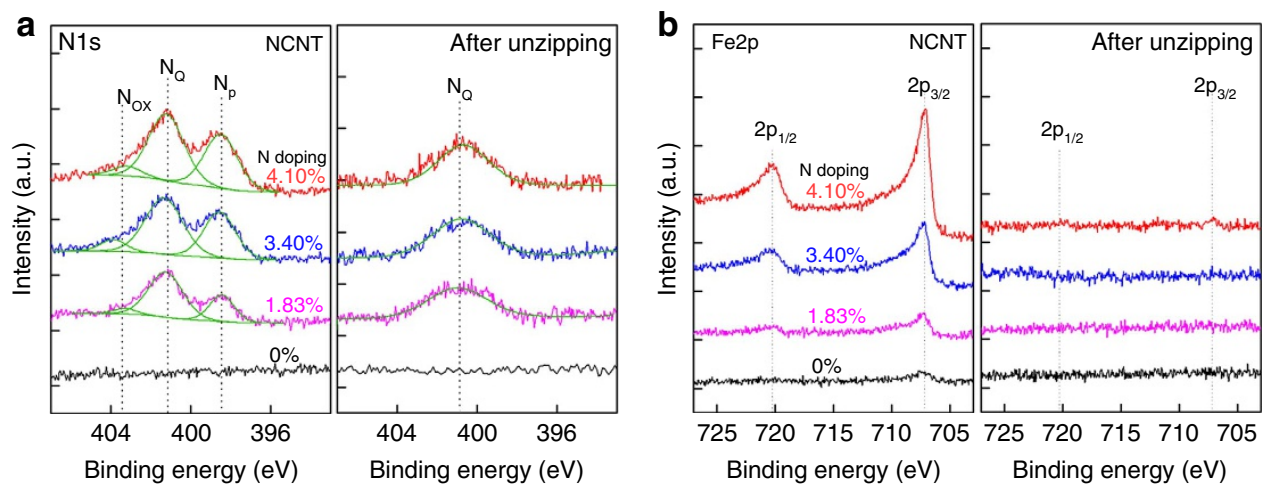

C
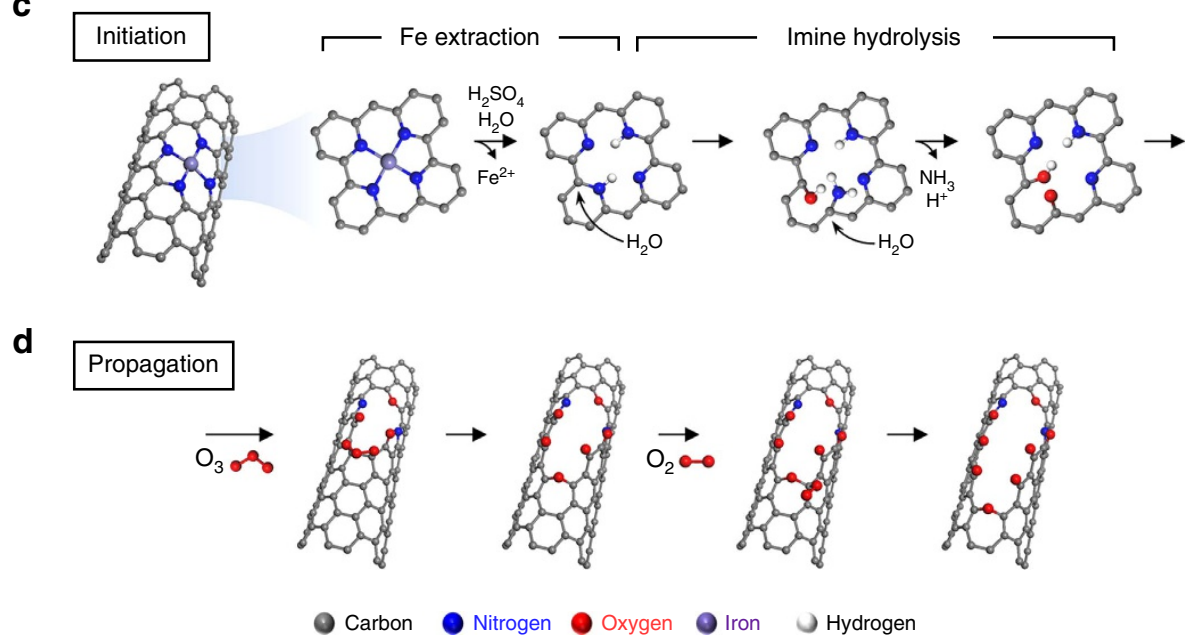

Figure 2 | Reaction mechanism for N-dopant-specific unzipping. (a,b) N1s and Fe2p XPS spectra before and after unzipping. $\mathrm{N}_{\mathrm{p}}$ (a) and Fe (b) completely disappear after unzipping. (c,d) Reaction mechanism for initiation and propagation stages. $\mathrm{Fe}^{2+}$ is extracted from FeN $\mathrm{N}_{4}$ site in NCNTs under acidic positivebiased condition, and subsequent acid-catalysed imine hydrolysis undergoes (c). Unzipping propagates along the longitudinal direction through continuous $\mathrm{C}=\mathrm{C}$ bond breaking with high-energy oxygen species, such as singlet $\mathrm{O}_{2}$ or $\mathrm{O}_{3}$ molecules (d).

Control of the unzipping time (at $0.8 \mathrm{~V}$ ) enables the fine controllability of unzipping propagation. Figure 3a shows TEM images of 2-, 8- and 16-h-unzipped structures. Although the outermost walls are selectively peeled off after $2 \mathrm{~h}$, fully unzipped structures are predominantly formed after $8 \mathrm{~h}$. Finally, completely torn small graphene flakes are observed after $16 \mathrm{~h}$. Interestingly, hexagonal crystallinity of graphitic domains remains intact in basal plane even after $16 \mathrm{~h}$. Figure $3 \mathrm{~b}$ presents the evolution of $\mathrm{O}$ and $\mathrm{N}_{\mathrm{p}}$ contents along with unzipping process. The drastic $\mathrm{N}_{\mathrm{p}}$ content reduction at the initial stage of unzipping substantiates the selective and concurrent commencement of unzipping initiation at multiple $\mathrm{N}_{\mathrm{p}}$-dopant sites, which are subsequently transformed into oxygen functional groups. The increasing rate of $\mathrm{O}$ or the decreasing rate of $\mathrm{N}_{\mathrm{p}}$ can be correlated with the overall unzipping rate. High unzipping rate rapidly diminishes for the first $8 \mathrm{~h}$ and gradually reaches to near-zero in the next $16 \mathrm{~h}$. The final structure obtained by prolonged 24-h-unzipping exhibits the $\mathrm{O}$ content of $11.9 \%$ along with the dramatic improvement in the dispersibility of unzipped structures in polar solvents ${ }^{39}$ (inset optical image in Fig. 3b and Supplementary Fig. 15).

Temporal evolution of capacitive current $\left(I_{c}\right)$ is another measure to monitor the unzipping progress, particularly in terms of surface area enhancement ${ }^{19} . I_{c}$ rapidly increases in the early stage because of the enlargement of surface area by gradual exposure of the inner surface of NCNTs, and the rate enhancement of $I_{c}$ slowdowns after $4 \mathrm{~h}$ (Fig. $3 \mathrm{c}$ ). The sudden reduction of $I_{\mathrm{c}}$ at $8 \mathrm{~h}$ reflects the serious damage of inmost wall of NCNTs that causes a loss of electrical connectivity ${ }^{40}$. Such a deterioration of the electrical integrity eventually leads to the self-termination of unzipping process.

Ultrahigh-power DLCs for AC filtering. As a typical application of our unique unzipping mechanism, AC-line filtering DLC is tested. Owing to the rapid electrical response through unscathed crystalline structure and the large surface area with vertical alignment, the unzipped forest structure surpasses conventional electrode materials for AC-line filtering, including metal foil or pristine graphitic carbons.

AC-line filtering DLC performance is analysed in a symmetric two-electrode configuration with identical unzipped forest electrodes over a $1-\mathrm{V}$ potential window in $1 \mathrm{M} \mathrm{KOH}$ solution without any binder or conducting additive. Cyclic voltammograms (CVs) were measured at $1-400 \mathrm{~V} \mathrm{~s}^{-1}$ (Fig. 4a). Reproducible rectangular shape curves were obtained even at $400 \mathrm{~V} \mathrm{~s}^{-1}$, indicating a near-ideal capacitive behaviour with stable electrical double layer formation and fast charge propagation throughout the electrodes. The ultrahigh-power performance is also illustrated by the linear dependence of discharge current upon scan rate as well as the little change in the areal capacitance as a function of scan rate (Supplementary Fig. 16a). This fast energy delivery capability was stably maintained up to 10,000 cycles, showing $96.2 \%$ of capacitance retention (Supplementary Fig. 16b)

Electrochemical impedance spectroscopy further confirms the outstanding power performance of the DLCs with unzipped 

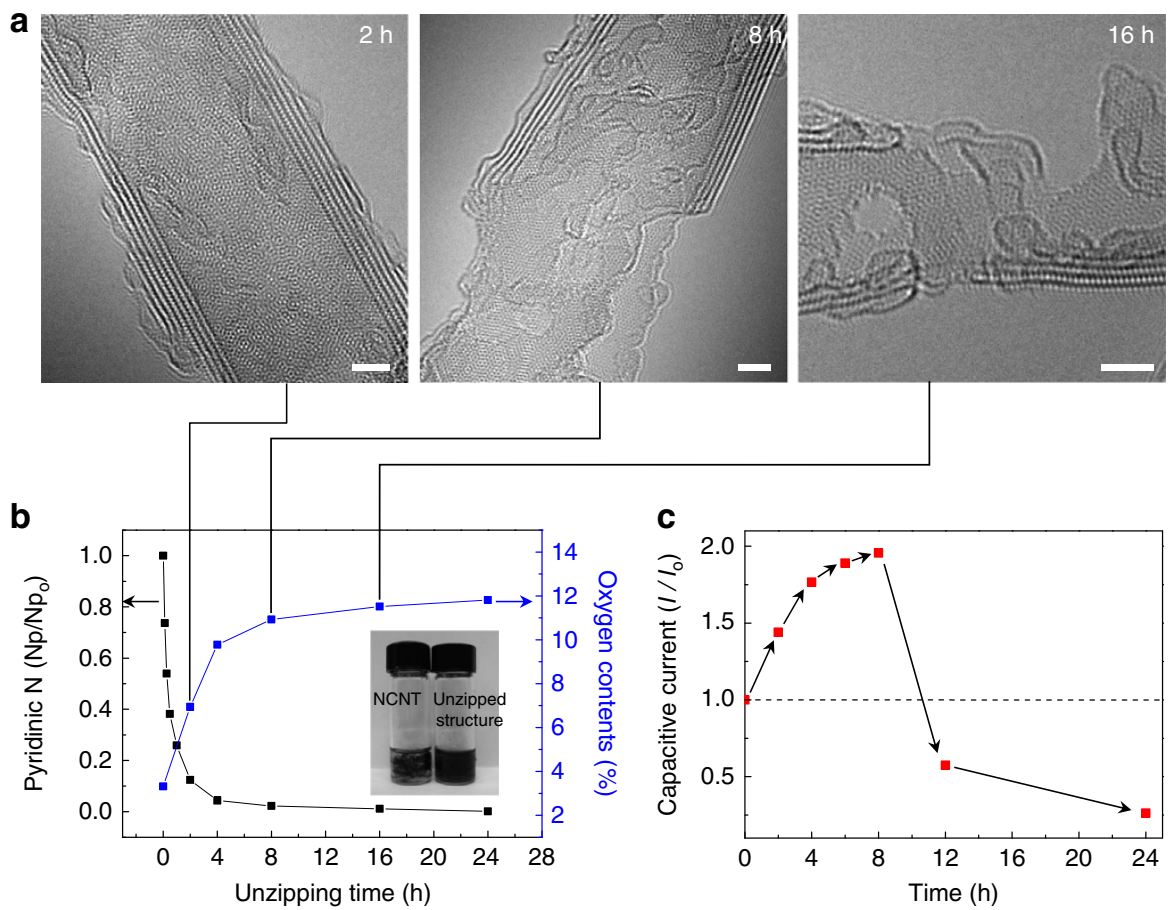

Figure 3 | Structure and chemical evolution during $\mathbf{N}$-dopant-specific unzipping of NCNTs. (a) Aberration-corrected TEM images of 2-, 8- and 16-hunzipped nanostructures (in $1 \mathrm{M} \mathrm{H}_{2} \mathrm{SO}_{4}$ at $0.8 \mathrm{~V}$ ). (b) $\mathrm{N}_{\mathrm{p}}$ and $\mathrm{O}$ contents versus unzipping time. (c) Capacitive current in cyclic voltammogram versus unzipping time. Scale bar, $2 \mathrm{~nm}$.

structures. Figure $4 \mathrm{~b}$ compares the impedance phase angles of DLCs at different frequencies for $1-\mu \mathrm{m}$ - and $5-\mu \mathrm{m}$-height unzipped forests, $5-\mu \mathrm{m}$-height pristine NCNT forest and typical aluminium electrolytic capacitor (AEC, $10 \mathrm{~V} / 220 \mu \mathrm{F}$ ). All tested DLCs show typical capacitive behaviours (near $-90^{\circ}$ phase angle) at $20 \mathrm{~Hz}$ but gradually evolve into resistive behaviours at higher frequencies. The DLCs made from $1-\mu \mathrm{m}$ - and 5 - $\mu \mathrm{m}$-height unzipped nanostructures reach $-45^{\circ}$ phase angle at 8.15 and $5.32 \mathrm{kHz}$, respectively. At $120 \mathrm{~Hz}$, a standard frequency for $\mathrm{AC}$ filter, the DLC with 1- $\mu \mathrm{m}$-unzipped structures shows approximately $-85^{\circ}$ of the impedance phase angle, which is comparable to that of AEC or other record-highperformance DLCs ${ }^{41-46}$. The DLC with 5- $\mu \mathrm{m}$-unzipped structures shows a slightly decreased impedance phase angle of $-78^{\circ}$ because of the elongated ion diffusion length and increased electrical resistance. The near-vertical line characteristics without semicircle and the low equivalent series resistance values from the Nyquist plots in Fig. 4c indicate that the unzipped nanostructure facilitates fast ion diffusion during charge/discharge operation.

In a DLC, series resistance, which hampers the power capability of the devices, can be simply considered as the sum of electronic and ionic resistance. The vertically aligned unzipped morphology of our electrode materials can effectively minimize the resistive elements. The vertical alignment offers idealized straight pathway for facile ion transport and homogeneous distribution of stored charges at electrode surfaces ${ }^{46,47}$. The intimate connection of the individual unzipped nanostructure to bottom current collector minimizes electronic resistance throughout the electrodes consisting of tightly attached CNT cores and unzipped nanostructures. Simultaneously, the exposure of inner CNT walls to the electrolyte and the improved wettability by hydrophilic oxygen-functionalized edges at unzipped structure allow for a greatly improved ion-accessibility to the electrode surfaces as well as the enhancement of accessible surface area ${ }^{44,48}$.

Specific areal capacitance $\left(C_{\mathrm{A}}, \mathrm{Fcm}^{-2}\right)$ is also calculated (equation (1) $)^{49}$. Owing to enlarged surface area, $5-\mu \mathrm{m}$-unzipped structure-based DLC exhibits $402 \mu \mathrm{Fcm}^{-2}$ at $120 \mathrm{~Hz}$, far higher than $230 \mu \mathrm{F} \mathrm{cm}^{-2}$ of the $5-\mu \mathrm{m}$-NCNT-DLC (Fig. 4d). Notably, $1-\mu \mathrm{m}$-unzipped structure-based DLC demonstrates $194 \mu \mathrm{F} \mathrm{cm}^{-2}$ at $120 \mathrm{~Hz}$, which is $\sim 84 \%$ of $5-\mu \mathrm{m}$-NCNT-DLC, and eventually outperform at a high frequency over $9 \mathrm{kHz}$. RC time constants $\left(\tau_{\mathrm{RC}}, \mathrm{R}\right.$ and $\mathrm{C}$ are total resistance and capacitance of devices, respectively) of DLCs fabricated with $5-\mu \mathrm{m}-\mathrm{NCNT}$ forest, 5- and 1- $\mu \mathrm{m}$-unzipped structures are 616,468 and $212 \mu$ s with 2.68 , 1.24 and $1.15 \Omega$ of total resistance at $120 \mathrm{~Hz}$, respectively. These values are sufficiently smaller than the standard $8.3 \mathrm{~ms}$ period, which is crucial for the effective AC line-filtering at $120 \mathrm{~Hz}$. The unzipped nanostructures also demonstrate a very small relaxation time constant $\left(\tau_{0}, 0.25 \mathrm{~ms}\right.$ for $1-\mu \mathrm{m}$-unzipped structure, Fig. $\left.4 \mathrm{e}\right)$ with moderate areal capacitance value, where $\tau_{0}$ is defined as the minimum time to discharge all the energy from the device with an efficiency greater than $50 \%$ (ref. 50). In the cases of DLCs with 5$\mu \mathrm{m}$-unzipped structure and $5-\mu \mathrm{m}-\mathrm{NCNT}, \tau_{0}$ increases to 0.36 and $1.22 \mathrm{~ms}$, respectively. These represent that the DLCs constructed with the customized unzipped structures show not only ultrahighpower performance, but also larger energy capacity at $120 \mathrm{~Hz}$. This finding illustrates that the unzipped nanostructures possess enormous potential for the rapid delivery of ultrahigh power.

\section{Discussion}

Unprecedented control of the graphene unzipping reaction is attained by substitutional heteroatom dopants. Highly specific unzipping initiation at $\mathrm{N}_{\mathrm{p}}$-dopant sites is confirmed by the significantly reduced electrochemical potential for unzipping and the distinctive correlation between unzipped morphology and $\mathrm{N}$-dopant density. It is noteworthy that $\mathrm{N}$-dopants have been suggested as readily oxidizable sites for the conventional chemical unzipping of $\mathrm{CNTs}^{51}$. Our work first reports a novel N-dopantspecific unzipping mechanism based on the systematic experimental characterization and theoretical analyses. Significantly, atomic-scale double-aberration corrected TEM 

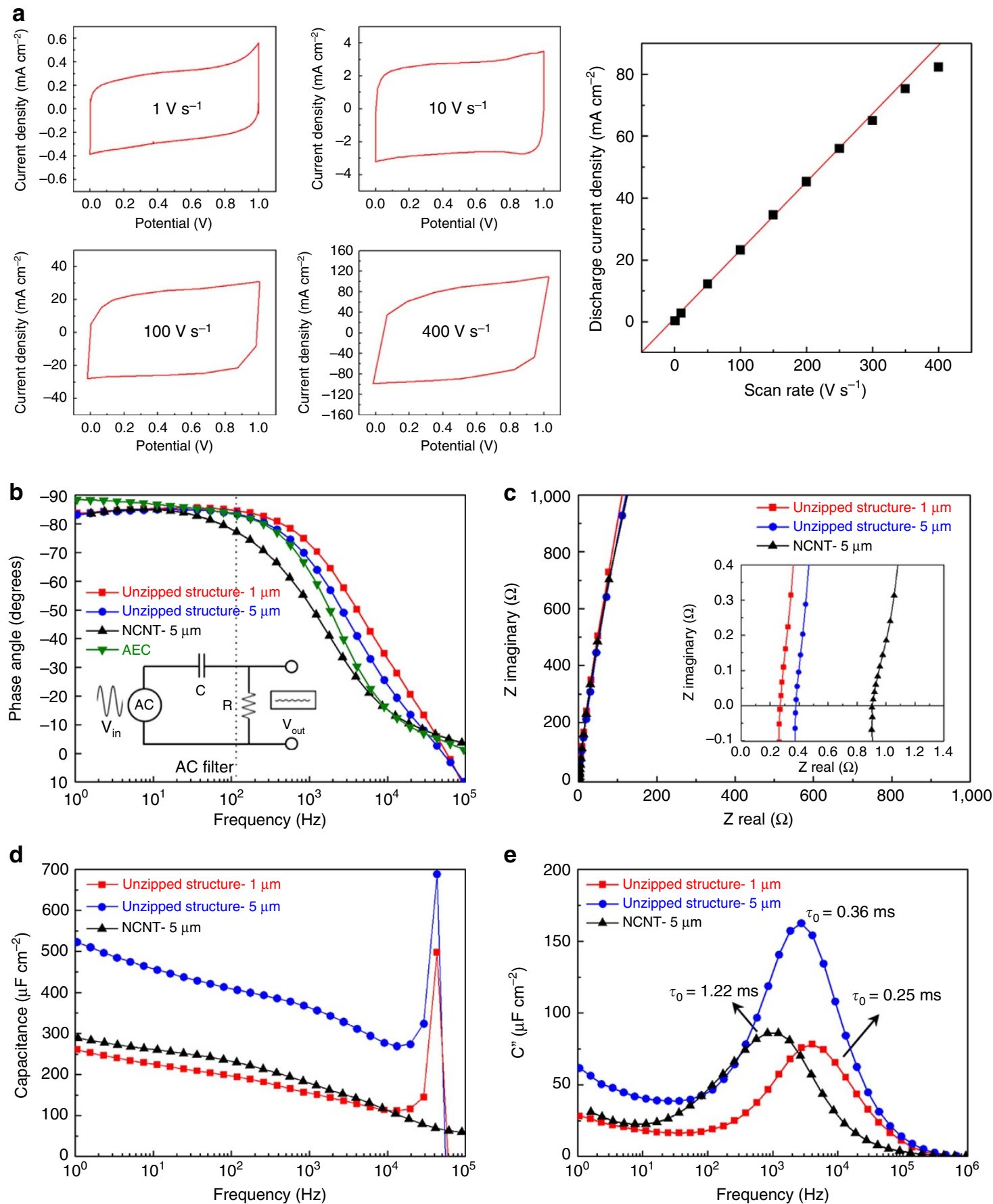

Figure 4 | Ultrahigh-power double layer capacitors with unzipped nanostructure forest electrodes. (a) Cyclic voltammograms at fast scan rates. (b) AC impedance phase angle versus frequency; vertical dotted line indicates $120 \mathrm{~Hz}$ frequency. (c) Complex plane plot of impedance; inset shows an expanded view in high-frequency region. (d) Specific capacitance versus frequency assuming a series RC circuit model. (e) Imaginary part (C") of specific capacitance versus frequency.

analysis confirms that this dopant-specific unzipping generates the graphene-based nanostructures with intact crystallinity and well-defined atomic configuration at unzipped edges. These unique features are feasible not only for multi-walled CNTs, but also for single-walled CNTs while maintaining the electrical connectivity through partially unzipped structures (Supplementary Fig. 17). Our novel reaction mechanism exploiting heteroatom dopants is clearly distinguishable from other preexisting unzipping methods, and addresses the key challenges, including the precise controllability of unzipping reaction and the preservation of intact crystalline graphene basal plane during unzipping process.

In this work, we report the ultrahigh-power performance of unzipped structure-based DLCs. In contrast to the intensive research efforts for high-energy-density supercapacitors ${ }^{15,52-55}$, power performance (or frequency response) enhancement of supercapacitors has attracted less research attention. Recently, ultrahigh-power supercapacitors capable of operating above $120 \mathrm{~Hz}$ have been newly recognized because of their possibility to replace the conventional AECs in AC filter circuit ${ }^{46}$. AECs 
Table 1 | A comparison of electrochemical performance of various carbon-based DLCs.

\begin{tabular}{|c|c|c|c|c|c|c|c|c|}
\hline Device & Reference & Materials & $\begin{array}{l}\text { Phase angle at } \\
120 \mathrm{~Hz}\left({ }^{\circ}\right)\end{array}$ & $\begin{array}{c}\tau_{\mathrm{RC}} \text { at } \\
120 \mathrm{~Hz} \\
(\mathrm{~ms})\end{array}$ & $\begin{array}{c}\text { Resistance at } \\
120 \mathrm{~Hz}(\Omega)\end{array}$ & $\begin{array}{c}\tau_{0} \\
(\mathrm{~ms})\end{array}$ & $\begin{array}{c}\mathrm{C}_{\mathrm{A}} \text { at } 120 \mathrm{~Hz} \\
\left(\mathrm{mF} \mathrm{cm}^{-2}\right)\end{array}$ & $\begin{array}{c}\mathrm{C}_{\mathrm{v}} \text { at } 120 \mathrm{~Hz} \\
\left(\mathrm{~F} \mathrm{~cm}^{-3}\right)\end{array}$ \\
\hline \multirow[t]{8}{*}{$\begin{array}{l}\text { Sandwich-type } \\
\text { device }\end{array}$} & This work & $\begin{array}{l}1 \mu \mathrm{m} \text {-Unzipped } \\
\text { nanostructure }\end{array}$ & 85 & 0.21 & 1.15 & 0.25 & 0.19 & $0.95^{*}$ \\
\hline & Miller et al. ${ }^{46}$ & VG & 82 & $\sim 0.2$ & 1.1 & NA & 0.09 & $0.73^{\star}$ \\
\hline & Sheng et al. ${ }^{45}$ & ErGO & 84 & 1.35 & 3.4 & 0.24 & 0.28 & $0.28^{\star}$ \\
\hline & Du and $\operatorname{Pan}^{57}$ & CNTs & $<75$ & NA & NA & 1.5 & NA & NA \\
\hline & T. Nathan-Walleser 58 & $\mathrm{TrGO}$ & 30 & 2.3 & NA & 4.1 & 3.6 & $1.8^{\star}$ \\
\hline & Pech et al. 50 & $A C$ & $\sim 1$ & NA & NA & 700 & NA & NA \\
\hline & El-kady et al. ${ }^{43}$ & $\mathrm{AEC}(63 \mathrm{~V} / 220 \mu \mathrm{F})$ & NA & NA & NA & NA & NA & $\sim 10^{-4}$ \\
\hline & Lin et al. ${ }^{44}$ & AEC & 83.9 & 0.14 & NA & NA & NA & NA \\
\hline \multirow[t]{3}{*}{ Micro-device } & Lin et al. ${ }^{44}$ & G/CNTCs & 81.5 & 0.20 & 21.3 & 0.82 & 0.23 & $0.23^{*}$ \\
\hline & El-kady et al. ${ }^{43}$ & LSG & $<30$ & NA & NA & $>1.6$ & NA & NA \\
\hline & Pech et al. ${ }^{50}$ & OLC & NA & NA & NA & 26 & NA & NA \\
\hline
\end{tabular}

AC, activated carbon; AEC, aluminium electrolytic capacitors; CNT, carbon nanotube; DLC, double-layer capacitor; ErGO, electrochemical reduced graphene oxide; G/CNTC, graphene-CNTs carpet; LSG: laser-scribed graphene; NA, not applicable; OLC, onion-like carbon; TrGO, thermally reduced graphene oxide; VG, vertical graphene.

All of the data are the best result in impedance phase angle in each literature.

"The values are estimated from the value of $C_{A}$ at $120 \mathrm{~Hz}$ and the thickness of only active material in each literature (equation (2))

are among the largest components in electronic circuit system that impede the reduction of circuit size, principally due to their low specific energy densities ${ }^{55}$. Supercapacitors with frequency response comparable to that of AECs are highly demanded for the effective miniaturization of electronic circuits and relevant portable devices. Our DLC with $1-\mu \mathrm{m}$-height unzipped structure exhibits rectangular shape CV curves even at the fast scan rate of $400 \mathrm{~V} \mathrm{~s}^{-1},-85^{\circ}$ of the impedance phase angle, $212 \mu \mathrm{s}$ of $\tau_{\mathrm{RC}}$ and $0.25 \mathrm{~ms}$ of $\tau_{0}$ at $120 \mathrm{~Hz}$. As summarized in Table 1, this remarkable power performance and AC frequency response are comparable or even superior to those of conventional AECs or the prime tier carbon nanomaterial-based devices reported thus far. Notably, the most proximate value to $-90^{\circ}$ in impedance phase angle and the lowest level of resistance $(1.15 \Omega)$ at $120 \mathrm{~Hz}$ indicate that the DLC with $1-\mu \mathrm{m}$-unzipped structure can properly function as well as typical AEC at $120 \mathrm{~Hz}$. Besides, our unzipped nanostructure electrode presents a volumetric capacitance $\left(C_{\mathrm{V}}=0.95 \mathrm{~F} \mathrm{~cm}^{-3}\right)$ at $120 \mathrm{~Hz}$, significantly higher than previous carbon based-electrodes. This $C_{\mathrm{V}}$ value is approximately three orders of magnitude higher than those of typical AECs $\left(\sim 10^{-4} \mathrm{~F} \mathrm{~cm}^{-3}\right)^{43,44,56}$.

As demonstrated from the record-high power DLCs, delicate manipulation of graphene-based nanostructures may offer straightforward routes to high-performance applications. The highly specific reaction features of our unzipping mechanism can serve as a generic principle to diversify graphene-based nanostructures, whose physicochemical properties are strongly dependent on their structural dimension and defect formation ${ }^{1-}$ 9. In principle, controlled cutting of $\mathrm{sp}^{2}$-hybridized carbon plane allows the transformation of structural dimension among zero-, one-, two- and three-dimensions. Overall, customized dopant-specific unzipping of graphene-based materials possibly guides us to rarely explored opportunity for multi-dimensional material design with atomic level precision as well as physicochemical property tuning, which are generally desired for energy storage, environmental remediation, nanomedicines and other versatile applications. Moreover, this precisely controllable unzipping mechanism grants a significant step forward to idealized intact crystalline graphene nanostructures in conjunction with the potential advances in the precise control of dopant position, dopant density and the chirality of CNTs.

\section{Methods}

Materials synthesis. NCNTs were grown by plasma-enhanced chemical vapour deposition with $\mathrm{Fe}$ catalyst. The density of $\mathrm{N}$ dopants was easily controllable by adjusting $\mathrm{NH}_{3}$ gas partial pressure and plasma power (Supplementary Fig. 12). As-grown vertical NCNT forest on $\mathrm{SiO}_{2} / \mathrm{Si}$ was floated on diluted hydrofluoric acid (HF) solution to separate NCNT forests from $\mathrm{SiO}_{2} / \mathrm{Si}$ substrate by wet-etching of $\mathrm{SiO}_{2}$ layer. The floated NCNT forests were carefully transferred onto a glassy carbon electrode. To enhance the mechanical and electrical contact between NCNT forests and glassy carbon electrode, the recovered electrode was thermally annealed at $65^{\circ} \mathrm{C}$ for $12 \mathrm{~h}$. The NCNT electrode was used as working electrode in typical three electrode configuration with mercury/mercury (I) sulfate reference electrode and platinum wire (counter electrode). The unzipping was carried out at room temperature in $1 \mathrm{M} \mathrm{H}_{2} \mathrm{SO}_{4}$ solution with positive electrical potential from 0.5 to $1.0 \mathrm{~V}$ for various unzipping period. Before each unzipping reaction, the electrolyte was purged with high purity $\mathrm{N}_{2}$ gas over $10 \mathrm{~min}$.

Materials characterization. HR-TEM images were collected on a doubleaberration corrected HR-TEM (Titan G2 60-300, FEI) located at KAIST Analysis Center for Research Advancement, operating with a monochromator (excitation $0.06)$ at $80 \mathrm{kV}$ accelerating voltage. The structural changes were also measured by field-emission scanning electron microscope (Magellan400, FEI). Samples were prepared by critical point drying to maintain the as-synthesized vertically aligned structure. AFM images were obtained with scanning probe microscope (MultiMode 8, Bruker). Samples were prepared by drop-casting from ethanol suspensions onto $\mathrm{SiO}_{2} / \mathrm{Si}$ substrate. Raman and XPS spectra were recorded with ARAMIS (Horiba Jobin Yvon) and Sigma probe (Thermo VG Scienfitic), respectively.

Electrochemical characterization. Unzipped nanostructures $\left(1 \mathrm{~cm}^{-2}\right)$ on $\mathrm{Pt}$ foil were used as an electrode for ultrahigh-power DLCs. Before DLC assembly, the unzipped structures were electrochemically reduced at $-0.6 \mathrm{~V}$ for $30 \mathrm{~min}$ to remove dispensable oxygen functional groups. The prototype DLCs were composed of two identical unzipped nanostructure electrodes and separator. All electrochemical characterization was carried out with a potentiostat (Bio-Logic, SP-200) using $1 \mathrm{M} \mathrm{KOH}$ solution as an electrolyte under ambient environment. All CVs in Fig. 4 were collected in 10th cycle at each scan rate. Electrochemical impedance spectroscopy measurements were conducted with sinusoidal signal of $10 \mathrm{mV}$ amplitude at various frequencies ranging from $10 \mathrm{mHz}$ to $1 \mathrm{MHz}$. A series-RC circuit model (equation (1)) was used to calculate the frequency response-specific areal capacitances $\left(C_{\mathrm{A}}, \mathrm{F} \mathrm{cm}^{-2}\right)$

$$
C_{\mathrm{A}}=\frac{-1}{2 \pi f S Z^{\prime \prime}(f)}
$$

where $f$ is frequency; $S$ is the total surface area of active materials region on both planar electrodes $\left(\mathrm{cm}^{2}\right) ; Z$ Z $(f)$ is the imaginary part of the impedance.

The frequency specific volumetric capacitances $\left(C_{\mathrm{V}}, \mathrm{F} \mathrm{cm}^{-3}\right)$ was calculated with equation (2)

$$
C_{\mathrm{V}}=\frac{C_{\mathrm{A}}}{d}
$$


where $C_{\mathrm{A}}$ is the specific areal capacitance calculated by equation (1); $d$ is total thickness of active material.

The specific capacitance of DLCs prepared with unzipped nanostructures could be calculated by using $C^{\prime}(f)$ and $C^{\prime \prime}(f)$,

$$
\begin{aligned}
& C^{\prime}(f)=\frac{-Z^{\prime \prime}(f)}{2 \pi f S|Z(f)|^{2}} \\
& C^{\prime \prime}(f)=\frac{Z^{\prime}(f)}{2 \pi f S|Z(f)|^{2}}
\end{aligned}
$$

where $C^{\prime}(f)$ is the real part of specific capacitance; $C^{\prime \prime}(f)$ is the imaginary part of specific capacitance; $Z^{\prime}(f)$ and $Z^{\prime \prime}(f)$ are real and imaginary parts of the impedance; $|Z(f)|$ is the absolute value of the impedance.

DFT calculation for unzipping mechanism. To understand the mechanism for electrochemical unzipping of CNTs, first-principles DFT calculation was performed using Vienna Ab-initio Simulation Package codes. The Perdew-BurkeEmzerhof exchange-correlation energy functional and projected-augmented wave potentials were employed. A plane-wave kinetic energy cutoff of $400 \mathrm{eV}$ and $1 \times 1 \times 1$ gamma k-point were used for 12 unit cells of $(5,5)$ single-walled CNT with 240 atoms. The supercell size is $20 \times 29.514 \times 20 \AA^{3}$ with the tube axis along the $y$ direction, and the diameter of model is about $6.726 \AA$. Atomic forces were relaxed $<0.025 \mathrm{eV}^{-1}$.

\section{References}

1. Kosynkin, D. V. et al. Longitudinal unzipping of carbon nanotubes to form graphene nanoribbons. Nature 458, 872-876 (2009).

2. Li, Y. et al. An oxygen reduction electrocatalyst based on carbon nanotubegraphene complexes. Nature Nanotech. 7, 394-400 (2012).

3. Huang, B., Son, Y.-W., Kim, G., Duan, W. \& Ihm, J. Electronic and magnetic properties of partially open carbon nanotubes. J. Am. Chem. Soc. 131, 17919-17925 (2009).

4. Tao, C. et al. Spatially resolving edge states of chiral graphene nanoribbons. Nature Phys. 7, 616-620 (2011).

5. Tapaszto, L., Dobrik, G., Lambin, P. \& Biro, L. P. Tailoring the atomic structure of graphene nanoribbons by scanning tunnelling microscope lithography. Nat. Nanotech. 3, 397-401 (2008).

6. Wang, X. et al. Graphene nanoribbons with smooth edges behave as quantum wires. Nat. Nanotech. 6, 563-567 (2011).

7. Lu, J., Yeo, P. S. E., Gan, C. K., Wu, P. \& Loh, K. P. Transforming $\mathrm{C}_{60}$ molecules into graphene quantum dots. Nat. Nanotech. 6, 247-252 (2011).

8. Ci, L. et al. Controlled nanocutting of graphene. Nano Res. 1, 116-122 (2008).

9. Liu, J. et al. Fullerene pipes. Science 280, 1253-1256 (1998).

10. Han, M., Özyilmaz, B., Zhang, Y. \& Kim, P. Energy band-gap engineering of gaphene nanoribbons. Phys. Rev. Lett. 98, 206805 (2007).

11. Wang, X. et al. Room-temperature all-semiconducting sub-10-nm graphene nanoribbon field-effect transistors. Phys. Rev. Lett. 100, 206803 (2008).

12. Jiao, L., Zhang, L., Wang, X., Diankov, G. \& Dai, H. Narrow graphene nanoribbons from carbon nanotubes. Nature 458, 877-880 (2009).

13. Shimizu, T. et al. Large intrinsic energy bandgaps in annealed nanotube-derived graphene nanoribbons. Nat. Nanotech. 6, 45-50 (2011).

14. Loh, K. P., Bao, Q., Ang, P. K. \& Yang, J. The chemistry of graphene. J. Mater. Chem. 20, 2277-2289 (2010).

15. Zhang, C. et al. Splitting of a vertical multiwalled carbon nanotube carpet to a graphene nanoribbon carpet and its use in supercapacitors. ACS Nano 7 , 5151-5159 (2013).

16. Higginbotham, A. L., Kosynkin, D. V., Sinitskii, A., Sun, Z. \& Tour, J. M. Lower-defect graphene oxide nanoribbons from multiwalled carbon nanotubes. ACS Nano 4, 2059-2069 (2010).

17. Cano-Márquez, A. G. et al. Ex-MWNTs: graphene sheets and ribbons produced by lithium intercalation and exfoliation of carbon nanotubes. Nano Lett. 9, 1527-1533 (2009).

18. Jiao, L., Wang, X., Diankov, G., Wang, H. \& Dai, H. Facile synthesis of highquality graphene nanoribbons. Nat. Nanotech. 5, 321-325 (2010).

19. Shinde, D. B., Debgupta, J., Kushwaha, A., Aslam, M. \& Pillai, V. K. Electrochemical unzipping of multi-walled carbon nanotubes for facile synthesis of high-quality graphene nanoribbons. J. Am. Chem. Soc. 133, 4168-4171 (2011).

20. Shinde, D. B., Majumder, M. \& Pillai, V. K. Counter-ion dependent, longitudinal unzipping of multi-walled carbon nanotubes to highly conductive and transparent graphene nanoribbons. Sci. Rep. 4, 4363 (2014).

21. Elías, A. L. et al. Longitudinal cutting of pure and doped carbon nanotubes to form graphitic nanoribbons using metal clusters as nanoscalpels. Nano Lett. 10, 366-372 (2009)

22. Morelos-Gómez, A. et al. Clean nanotube unzipping by abrupt thermal expansion of molecular nitrogen: graphene nanoribbons with atomically smooth edges. ACS Nano 6, 2261-2272 (2012).
23. Ozden, S. et al. Unzipping carbon nanotubes at high impact. Nano Lett. 14, 4131-4137 (2014).

24. Hossain, M. Z. et al. Chemically homogeneous and thermally reversible oxidation of epitaxial graphene. Nat. Chem. 4, 305-309 (2012).

25. Terrones, M. Sharpening the chemical scissors to unzip carbon nanotubes: crystalline graphene nanoribbons. ACS Nano 4, 1775-1781 (2010).

26. John, R. et al. Sequential electrochemical unzipping of single-walled carbon Nanotubes to graphene ribbons revealed by in situ raman spectroscopy and imaging. ACS Nano 8, 234-242 (2013).

27. Terrones, M. Materials science: nanotubes unzipped. Nature 458, 845-846 (2009).

28. Ajayan, P. M. \& Yakobson, B. I. Material science: oxygen breaks into carbon world. Nature 441, 818-819 (2006).

29. Ritter, K. A. \& Lyding, J. W. The influence of edge structure on the electronic properties of graphene quantum dots and nanoribbons. Nat. Mater. 8, 235-242 (2009).

30. Nakada, K., Fujita, M., Dresselhaus, G. \& Dresselhaus, M. S. Edge state in graphene ribbons: Nanometer size effect and edge shape dependence. Phys, Rev. B 54, 17954 (1996).

31. Fan, Z.-J. et al. Facile synthesis of graphene nanosheets via Fe reduction of exfoliated graphite oxide. ACS Nano 5, 191-198 (2011).

32. Jin, Z. et al. Ultralow feeding gas flow guiding growth of large-scale horizontally aligned single-walled carbon nanotube arrays. Nano Lett. 7, 2073-2079 (2007).

33. Rao, R., Liptak, D., Cherukuri, T., Yakobson, B. I. \& Maruyama, B. In situ evidence for chirality-dependent growth rates of individual carbon nanotubes. Nat. Mater. 11, 213-216 (2012).

34. Lee, D., Lee, W., Lee, W., Kim, S. \& Kim, Y.-H. Theory, synthesis, and oxygen reduction catalysis of Fe-porphyrin-like carbon nanotube. Phys. Rev. Lett. 106, 175502 (2011)

35. Li, J.-L. et al. Oxygen-driven unzipping of graphitic materials. Phys. Rev. Lett. 96, 176101 (2006).

36. Gong, K., Du, F., Xia, Z., Durstock, M. \& Dai, L. Nitrogen-doped carbon nanotube arrays with high electrocatalytic activity for oxygen reduction. Science 323, 760-764 (2009).

37. Lee, D. H., Lee, W. J. \& Kim, S. O. Highly efficient vertical growth of wallnumber-selected, N-doped carbon nanotube arrays. Nano Lett. 9, 1427-1432 (2009).

38. Debgupta, J., Shinde, D. B. \& Pillai, V. K. In situ electrochemical organization of CdSe nanoclusters on graphene during unzipping of carbon nanotubes. Chem. Comm. 48, 3088-3090 (2012).

39. Kotov, N. A. Materials science: carbon sheet solutions. Nature 442, 254-255 (2006).

40. Maiti, U. N. et al. 25th Anniversary Article: Chemically modified/doped carbon nanotubes \& graphene for optimized nanostructures \& nanodevices. Adv. Mater. 26, 40-67 (2014).

41. Lee, J. A. et al. Ultrafast charge and discharge biscrolled yarn supercapacitors for textiles and microdevices. Nat. Commun. 4, 1970 (2013).

42. Wang, X. et al. Three-dimensional strutted graphene grown by substrate-free sugar blowing for high-power-density supercapacitors. Nat. Commun. 4, 2905 (2013).

43. El-Kady, M. F. \& Kaner, R. B. Scalable fabrication of high-power graphene micro-supercapacitors for flexible and on-chip energy storage. Nat. Commun. 4, 1475 (2013).

44. Lin, J. et al. 3-dimensional graphene carbon nanotube carpet-based microsupercapacitors with high electrochemical performance. Nano Lett. 13, 72-78 (2012)

45. Sheng, K., Sun, Y., Li, C., Yuan, W. \& Shi, G. Ultrahigh-rate supercapacitors based on eletrochemically reduced graphene oxide for ac line-filtering. Sci. Rep. 2, 247 (2012).

46. Miller, J. R., Outlaw, R. A. \& Holloway, B. C. Graphene double-layer capacitor with ac line-filtering performance. Science 329, 1637-1639 (2010).

47. Futaba, D. N. et al. Shape-engineerable and highly densely packed single-walled carbon nanotubes and their application as super-capacitor electrodes. Nature Mater. 5, 987-994 (2006).

48. Sinani, V. A. et al. Aqueous dispersions of single-wall and multiwall carbon nanotubes with designed amphiphilic polycations. J. Am. Chem. Soc. 127, 3463-3472 (2005).

49. Taberna, P., Simon, P. \& Fauvarque, J.-F. Electrochemical characteristics and impedance spectroscopy studies of carbon-carbon supercapacitors. $J$. Electrochem. Soc. 150, 292-300 (2003).

50. Pech, D. et al. Ultrahigh-power micrometre-sized supercapacitors based on onion-like carbon. Nat. Nanotech. 5, 651-654 (2010).

51. Cruz-Silva, R. et al. Formation of nitrogen-doped graphene nanoribbons via chemical unzipping. ACS Nano 7, 2192-2204 (2013).

52. Wu, Z.-S. et al. High-energy $\mathrm{MnO}_{2}$ nanowire/graphene and graphene asymmetric electrochemical capacitors. ACS Nano 4, 5835-5842 (2010).

53. Wu, Z. S. et al. Anchoring hydrous $\mathrm{RuO}_{2}$ on graphene sheets for highperformance electrochemical capacitors. Adv. Func. Mater. 20, 3595-3602 (2010). 
54. Zhang, K., Zhang, L. L., Zhao, X. \& Wu, J. Graphene/polyaniline nanofiber composites as supercapacitor electrodes. Chem. Mater. 22, 1392-1401 (2010).

55. Maiti, U. N., Lim, J., Lee, K. E., Lee, W. J. \& Kim, S. O. Three-dimensional shape engineered, interfacial gelation of reduced graphene oxide for high rate, large capacity supercapacitors. Adv. Mater. 26, 615-619 (2014).

56. Pandolfo, A. G. \& Hollenkamp, A. F. Carbon properties and their role in supercapacitors. J. Power Sources 157, 11-27 (2006).

57. Du, C. \& Pan, N. Supercapacitors using carbon nanotubes films by electrophoretic deposition. J. Power Sources 160, 1487 (2006).

58. Nathan-Walleserl, T. et al. 3D Micro-extrusion of graphene-based active electrodes: towards high-rate AC line filtering performance electrochemical capacitors. Adv. Func. Mater. 24, 4706-4716 (2014).

\section{Acknowledgements}

This work was supported by the Multi-Dimensional Directed Nanoscale Assembly Creative Research Initiative (CRI) Center (2015R1A3A2033061) of the National Research Foundation of Korea (MSIP).

\section{Author contributions}

J.L. principally performed the experiments and leaded this project. S.O.K. supervised the entire project. S.O.K., Y-.H.K., J.L. and W.J.L. conceived the idea and designed the overall experiments. J.L., U.N.M. and J.M.L. carried out the materials synthesis, characterizations and fabrication of double layer capacitors. Y.-H.K. and N.-Y.K. carried out the DFT calculations and analysed the results. D.S.C., G.Y.L. and S.H.K.

performed X-ray photoelectron spectroscopy and Raman spectroscopy measurements, and analysed the results. Y-.H.K., H.K., R.N., N-.Y.K. and Y.O. undertook the study for reaction mechanism. All authors co-wrote the paper and participated in discussions.

\section{Additional information}

Supplementary Information accompanies this paper at http://www.nature.com/ naturecommunications

Competing financial interests: The authors declare no competing financial interests.

Reprints and permission information is available online at http://npg.nature.com/ reprintsandpermissions/

How to cite this article: Lim, J. et al. Dopant-specific unzipping of carbon nanotubes for intact crystalline graphene nanostructures. Nat. Commun. 7:10364 doi: $10.1038 /$ ncomms10364 (2016).

(c) (1) article are included in the article's Creative Commons license, unless indicated otherwise in the credit line; if the material is not included under the Creative Commons license, users will need to obtain permission from the license holder to reproduce the material. To view a copy of this license, visit http://creativecommons.org/licenses/by/4.0/ 